\title{
Histórico e evolução do conhecimento sobre a paleoflora do Devoniano na Bacia do Paraná
}

\author{
History and evolution of knowledge about Devonian \\ palaeoflora of Paraná Basin
}

\author{
Historia y evolución del conocimiento sobre la paleoflora del \\ Devónico de la cuenca Paraná
}

\author{
William Mikio Kurita Matsumura \\ williammatsumura@gmail.com \\ Universidade Federal do Rio Grande do Sul
}

Roberto Iannuzzi

roberto.iannuzzi@ufrgs.br Universidade Federal do Rio Grande do Sul

Elvio Pinto Bosetti

elvio.bosetti@pq.cnpq.br

Universidade Estadual de Ponta Grossa

\begin{abstract}
Resumo: Nos últimos anos, a execução de trabalhos de campo no município de TibagiPR tem possibilitado a prospecção de fitofósseis bem preservados na Formação São Domingos (Bacia do Paraná), resgatando o interesse científico da paleobotânica nesta unidade. O presente trabalho objetivou caracterizar a evolução do conhecimento sobre a paleoflora devoniana da Bacia do Paraná, com base no histórico das pesquisas no Brasil e apresentar as perspectivas futuras a partir de novos achados em seções recentementelevantadas do Devoniano Médiono estado do Paraná. Foramidentificados e descritos três táxons ocorrentes nos afloramentos Sítio Wolff e Itáytyba (Formação São Domingos), a saber: Spongiophyton, Palaeostigma e Haplostigma. A continuidade das pesquisas com este material fóssil pode colaborar na compreensão dos estudos evolutivos e paleoecológicos das plantas, além de fornecer dados relevantes para interpretações dos sistemas deposicionais e reconstituições paleofitogeográficas.
\end{abstract}

Palavras-chave: Paleobotânica. Bacia do Paraná. Formação São Domingos. Devoniano.

\begin{abstract}
Currently, the fieldwork developed in the Tibagi municipality, Paraná state, have enabled the prospection of phytofossils preserved in the São Domingos Formation (Paraná Basin), bringing back the scientific interest of paleobotany on this unit. The present study aimed to characterize the evolution of knowledge about Devonian paleoflora of the Paraná Basin, based on the history of research in Brazil and present future perspectives from new findings in sections recently described in the Middle
\end{abstract}


Devonian of Paraná state. Spongiophyton, Palaeostigma and Haplostigma were identified and described from Sítio Wolff and Itáytyba outcrops (São Domingos Formation). Further studies with this fossil material can contribute to the knowledge development in the evolutionary and paleoecological studies of plants, besides providing data relevant to interpretation of depositional systems and palaeophytogeographical reconstructions.

Keywords: Paleobotany. Paraná basin. São Domingos Formation. Devonian.

Resumen: En los últimos años, los trabajos de campo en la ciudad de Tibagi (PR) han permitido la prospección de fósiles vegetales en la Formación São Domingos (Cuenca Paraná), y los hallazgos realizados hasta el momento ampliaron el conocimiento paleobotánico de esta unidad. Este trabajo tiene como objetivo caracterizar la evolución del conocimiento sobre la paleoflora devónica de la Cuenca Paraná, con base en la historia de las investigaciones en Brasil y presentar las perspectivas de futuro a partir de nuevos hallazgos en secciones inéditas del Devónico Médio de la cuenca. Fueron identificados y descripto tres taxones ocurrentes en afloramientos Sítio Wolff e Itáytyba (Formación São Domingos), a saber: Spongiophyton, Palaeostigma y Haplostigma. La continuidad de las investigaciones sobre este material fósil permitirá mejorar aspectos taxonómicos relacionados a dichos taxones y contribuirá en el análisis evolutivo y aspectos paleoecológicos de las plantas del Devónico Medio, además de proporcionar datos para la interpretación de los sistemas depositacionales y reconstrucciones paleofitogeográficas.

Palabras clave: Paleobotánica. Cuenca Paraná. Formación São Domingos. Devónico.

\section{INTRODUÇÃO}

A paleoflora da sucessão devoniana do estado do Paraná é composta por fragmentos de plantas vasculares (Tracheophyta) e avasculares (Bryophyta), ocorrentes na porção superior da Formação Furnas (Eolochkoviano), talos algálicos na Formação Ponta Grossa (Neopraguiano/Eoemsiano) e fragmentos de Spongiophyton sp. (Spongiophytaceae), Palaeostigma sp. (Tracheophyta) e Haplostigma sp. (Lycopsida) na Formação São Domingos (Neoemsiano/Frasniano) (sensu GAUGRIS; GRAHN, 2006; MENDLOWICZ MAULLER et al., 2009; GRAHN et al., 2011) (Figura 1).

Grande parte do interesse nestes grupos reside no fato de que eles registram os primeiros estágios da evolução das plantas vasculares primitivas adaptadas ao ambiente terrestre e sua história de ocupação na Bacia do Paraná. Apesar dos vários trabalhos existentes na literatura especializada sobre paleobotânica do Devoniano da Bacia do Paraná (e.g. SOMMER, 1954; KRÄUSEL, 1954; GUERRA-SOMMER 1993; GUERRASOMMER et al., 1996; MUSSA et al., 1996; GERRIENNE et al., 2001; MILAGRES et al., 2007; MACHADO et al., 2007), estes ainda são em muito menor número do que aqueles referentes às paleofaunas (CLARKE, 1913; BOUCOT; GILL, 1956; BOUCOT, 1971; BALDIS, 1979; MELO, 1988; QUADROS, 1987; SIMÕES et al., 2002; BOSETTI, 2004; SCHEFFLER, 
2007; 2010; SCHEFFLER; FERNANDES, 2007a; 2007b; BOSETTI et al., 2009; 2011; 2012; ZABINI et al., 2010; 2012, dentre muitos outros), principalmente, se considerarmos apenas aqueles que abordam as paleofloras das formações Ponta Grossa (SOMMER, 1954) e São Domingos (MATSUMURA et al., 2010; 2011; 2012; 2013).

O processo de colonização inicial dos continentes pelas plantas vasculares durante o Siluro-Devoniano vem sendo descrito apenas a partir de análises de materiais fossilíferos obtidos na Laurussia ou Euramerica, Norte e Leste do Gondwana (RAYMOND et al., 1985; RAYMOND, 1987; EDWARDS, 1990). Só mais recentemente, este registro paleobotânico vem sendo reconhecido e estudado de forma sistemática na região Oeste do Gondwana (GERRIENNE et al., 2001; MILAGRES et al., 2007). Nas últimas duas décadas, as descobertas de plantas vasculares devonianas em diferentes localidades da América do Sul [Brasil (Eodevoniano, GERRIENNE et al., 2001), Uruguai (Eodevoniano, SPRECHMANN et al., 1993), Bolívia (Eo/Mesodevoniano, Di PASQUO; NOETINGER, 2008; RACHEBOEUF et al., 2012), Colômbia (Mesodevoniano, BERRY et al., 2000), Argentina (Mesodevoniano, CINGOLANI et al., 2002; Di PASQUO et al., 2009), Venezuela (Meso/Neodevoniano, BERRY \& EDWARDS, 1995) e Chile (Neodevoniano, MOISAN et al., 2011)] que integravam o Gondwana Ocidental, têm aberto novas possibilidades de aprofundamento e de revisões sobre pontos importantes da história inicial da evolução dessas plantas primitivas neste continente. Por exemplo, sabe-se que, diferentemente das províncias paleofitogeográficas contemporâneas situadas nas médias a baixas latitudes (Domínios Angárico, Euramericano e Norte do Gondwana, RAYMOND et al., 1985; RAYMOND, 1987; WNUK, 1996), cujos primeiros registros datam do Neossiluriano, a flora que ocupava o Gondwana Ocidental, sobretudo na Bacia do Paraná, teve o seu início apenas no Eodevoniano. Em adição, a flora do Gondwana meridional de altas latitudes $\left(70-80^{\circ} \mathrm{S}\right)$ apresentava-se pouco diversificada, com possível grau de endemismo e sujeita a condições ambientais restritivas (GERRIENNE et al., 2001).

Assim, os modelos paleogeográficos, paleoclimáticos e paleoambientais vêm sendo reinterpretados em função da agregação de novas informações (MILAGRES et al., 2007), sobretudo, no Mesodevoniano da Bacia do Paraná (Di PASQUO et al., 2009). O presente trabalho objetiva caracterizar a evolução do conhecimento sobre a paleoflora devoniana do estado do Paraná, com base no histórico das pesquisas no Brasil e apresentar as perspectivas a partir de novos achados em seções recentemente levantadas do Devoniano Médio no Paraná. 
Figura 1. A-B. Mapa de localização das seções devonianas da Bacia do Paraná (Sub-bacia de Apucarana) contendo plantas terrestres. 1-2. Formação Furnas (GERRIENNE et al., 2001; MILAGRES et al., 2007); 3. Formação Furnas (RODRIGUES et al., 1989); 4. Formação Furnas (BOLZON et al., 1994; MACHADO et al., 2007); 5. Formação Furnas (BIGARELLA et al., 1966); 6. Formação São Domingos (KRÄUSEL, 1954; OPPENHEIM, 1935); 7. Formação São Domingos (BOSETTI et al., 2011); 8. Formação São Domingos (MATSUMURA et al., 2010; 2011; 2013)
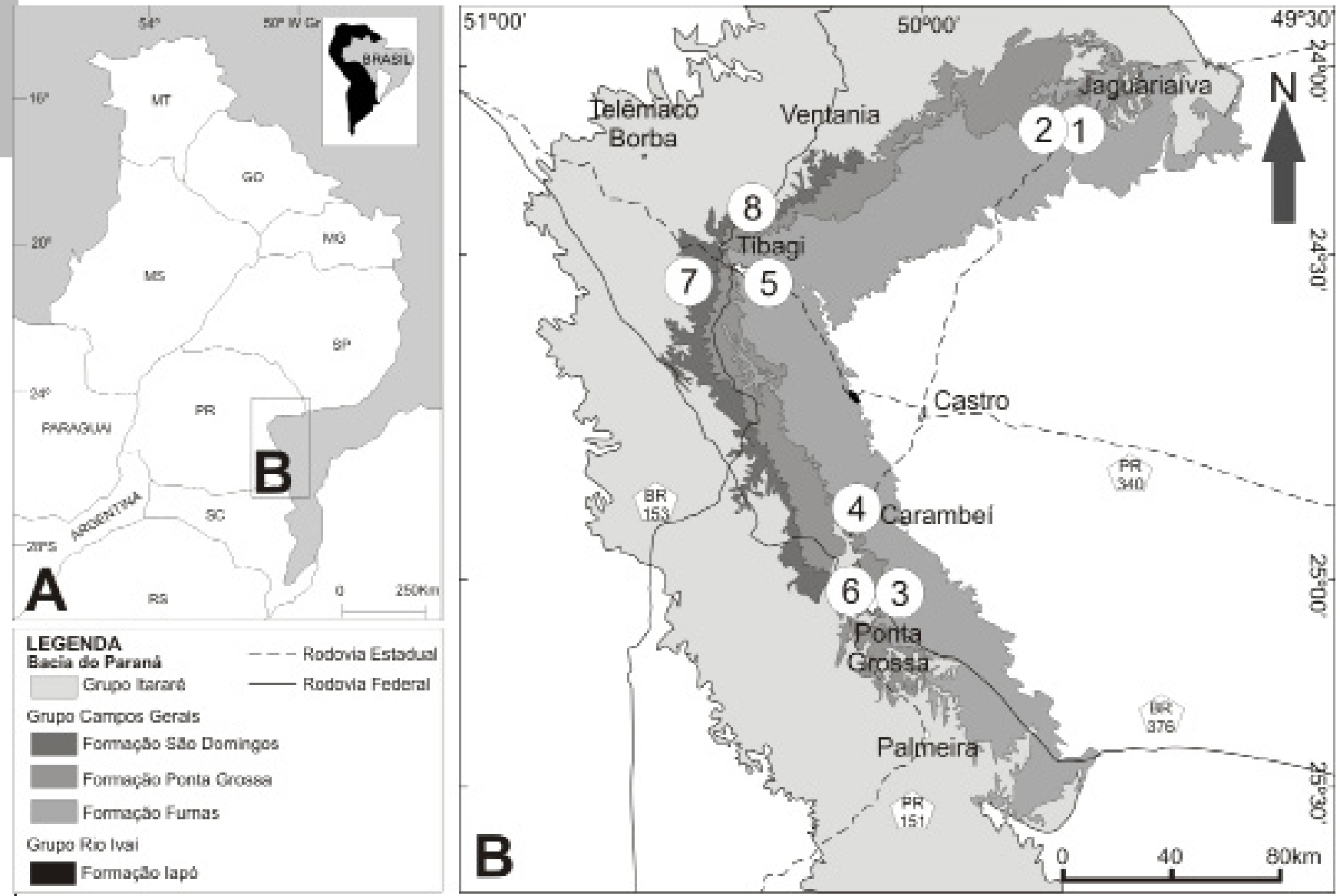

\section{HISTÓRICO DAS PESQUISAS E EVOLUÇÃO DO CONHECIMENTO DA PALEOFLORA DEVONIANA DO ESTADO DO PARANÁ}

A primeira descrição de fragmentos de plantas fósseis devonianas ocorrentes no estado do Paraná foi realizada por Clarke (1913). O material, proveniente do município de Ponta Grossa-PR, foi atribuído como "Problematum" pelo autor. Já Oppenheim (1935) determinou os restos vegetais encontrados em Ponta Grossa, como fragmentos de Lepidodendron e Calamites. Read (1941) mencionou, sem descrição, a presença de Psilophyton sp. no mesmo município. Essas pesquisas pioneiras correspondem à primeira fase da evolução do conhecimento do Devoniano do estado do Paraná (final do século XIX e início do século XX) proposta por Bosetti et al. (2007). Esta fase é caracterizada pelas intensas análises descritivas e classificatórias que refletiram as tendências da História Natural daquela época.

A partir de 1940 até 1980, a segunda fase de estudos é promovida, e nela se buscou por respostas a questionamentos de caráter interpretativo, onde foram abordados temas e proposições de modelos envolvendo a autoctonia ou aloctonia dos fósseis, seu 
aparente endemismo e, principalmente, suas relações com outras bacias sedimentares contemporâneas (BOSETTI et al., 2007). São desta fase os trabalhos de Barbosa (1949), Sommer (1954), Kräusel (1954), Guerra-Sommer (1993), Guerra-Sommer et al. (1990; 1996).

Barbosa (1949) classificou um vegetal encontrado em Jaguariaíva-PR, como Orvillea petrii gen. e sp. nov., enquanto outros restos, provenientes de Ponta Grossa e da Bolívia (La Torre), foram atribuídos ao gênero Haplostigma erejido por Seward (1932) para material proveniente do Devoniano da África do Sul, tendo sido classificados como Haplostigma lenticularis sp. nov. e Haplostigma irregularis. Em 1954, Sommer redescreveu o material "Problematum" de Clarke (1913) e Orvillea petrii de Barbosa (1949), propondo Euzebiola clarkei e Octaviona petrii, respectivamente.

Kräusel (1954), ao analisar o material fóssil proveniente de Ponta Grossa cedido pelo paleontólogo Frederico Waldemar Lange (1911-1988), descreveu um novo gênero de planta para o Devoniano paranaense, i.e. Spongiophyton gen. nov. O autor ainda redescreveu Haplostigma lenticularis de Barbosa (1949) como Spongiophyton lenticulare, reconhecendo também as espécies $S$. nanum, S. minutissimum, S. articulatum e $S$. hirsutum para esse novo gênero, além de Haplostigma irregularis (Schwarz) Seward, 1932. Posteriormente, o gênero Spongiophyton foi reconhecido na bacia sedimentar do Parnaíba, no Brasil (KRÄUSEL \& DOLIANITI, 1957), além de Gana (CHALONER et al., 1974), Canadá (GENSEL et al., 1991), Polônia (ZDEBSKA, 1978), Bolívia (BOUREAU \& PONS, 1973) e Marrocos (GERRIENNE et al., 1999). Em seguida, Kräusel \& Venkatachala (1966), ao analisarem o material proveniente do Devoniano da Sibéria, caracterizaram o novo gênero Aculeophyton que englobaria a espécie Spongiophyton hirsutum de Kräusel (1954).

Guerra-Sommer et al. (1990) identificaram Spongiophyton associados a trilobitas e icnofósseis em estratos da Formação São Domingos no estado do Paraná. Guerra-Sommer (1993), ao analisar a distribuição de Spongiophyton nas bacias do Paraná e Parnaíba, realizou considerações de caráter paleogeográfico, paleoambiental e bioestratigráfico para o gênero. Guerra-Sommer et al. (1996), tendo realizado estudos morfológicos, geoquímicos e petrográficos, propuseram o hábito terrestre para Spongiophyton. Deste modo, os estudos realizados por Guerra-Sommer et al. (1996) contribuíram para o avanço do conhecimento sobre este fóssil enigmático.

Mais recentemente, Matsumura et al. (2010) e Bosetti et al. (2011) registraram a ocorrência de fragmentos vegetais (Spongiophyton sp.) e realizaram breves descrições da ocorrência de impressões e compressões fósseis bem preservadas de Spongiophyton sp. e Lycopsida na Formação São Domingos em Tibagi.

Apesar dos primeiros achados de fósseis vegetais terem sido provenientes dos estratos das formações Ponta Grossa e São Domingos, as pesquisas paleobotânicas se concentraram no estudo das plantas vasculares terrestres ocorrentes na Formação Furnas em diversas localidades do Paraná. Petri (1948) registrou fósseis vegetais no topo da Formação Furnas, nos municípios de Ponta Grossa e Tibagi. Bigarella et al, (1966) descreveram a ocorrência de fragmentos vegetais em afloramento de topo da Formação Furnas, no município de Tibagi. Rodrigues et al. (1989) registraram a ocorrência de Psilophytales em afloramentos da Formação Furnas, no município de Ponta Grossa. Mussa; Borghi (1993) 
analisaram impressões do gênero Horneophyton e outras formas inéditas em diversos afloramentos em Jaguariaíva. Mussa et al. (1996) e Mussa et al. (2002) identificaram novos táxons de esporófitos (Salopella brasiliana e Sphaerullophyton originalis) e de gametangióforos (Edwardsnella campanulata), além de eixos estéreis com ramificações dicotômicas (Sulculiphyton furnasensis) encontrados em afloramentos da Formação Furnas, também em Jaguariaíva.

Bolzon et al. (1994) registraram a primeira ocorrência do gênero Cooksonia no topo da Formação Furnas, no Paraná. Gerrienne et al. (2001) descreveram uma nova espécie de Cooksonia, C. paranensis e outras formas de plantas terrestres primitivas (Cooksonia cf. cambrensis; Pertonella sp. A; Tarrantia sp.; Sporogonites sp. A; e mais dois espécimes sem identificação) provenientes de duas localidades (Jackson Figueiredo e PISA) da Formação Furnas em Jaguariaíva. Posteriormente, Gerrienne et al. (2006) sugeriram um ciclo de vida heteromórfico e de dominância esporofítica para o gênero Cooksonia, considerando-o junto com outras Eutracheophyta primitivas, ancestral de todas as plantas vasculares modernas.

Machado et al. (2007) descreveram, em afloramentos da Formação Furnas em Carambeí, a morfologia de esporângios atribuídos a Cooksonia paranaenses e Cooksonia sp., além de esporângios e eixos estéreis indeterminados. Milagres et al. (2007) registraram novas ocorrências de vegetais fósseis para o topo da Formação Furnas nas localidades de Jackson Figueiredo e PISA, em Jaguariaíva, abordando questões sobre a evolução e dispersão das plantas vasculares primitivas. Gonez \& Gerrienne (2010) assinalaram a presença de Aberlemnia caledonica para a Formação Furnas.

Por fim, segundo Bosetti et al. (2007), a partir de 1989, a análise de feições tafonômicas é utilizada com frequência cada vez maior para a interpretação do registro fossilífero. Porém, foi somente a partir do final da década de 1990 que trabalhos de cunho estritamente tafonômicos começaram a surgir na literatura. No entanto, grande parte dos estudos com abordagem tafonômica/estratigráfica desenvolvidos na região (e.g. SIMÕES et al., 2002; SIMÕES et al., 2003; RODRIGUES et al., 2001; RODRIGUES, 2002; GHILARDI, 2004; BOSETTI et al., 2009; 2011; 2012; ZABINI et al., 2010; 2012) foram executados com as macropaleofauna de invertebrados marinhos. O estudo tafonômico do material paleobotânico da sucessão devoniana do Paraná tem sido recentemente desenvolvido (MATSUMURA et al., 2013) de forma preliminar, ilustrando a lacuna existente nesta área.

\section{MATERIAL E MÉTODOS}

O material paleontológico é proveniente dos afloramentos Sítio Wolff (Seção colunar

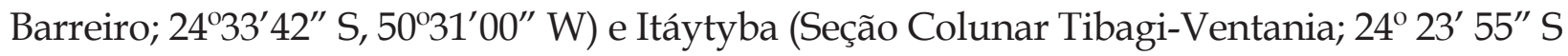
$\left.50^{\circ} 20^{\prime} 16^{\prime \prime} \mathrm{W}\right)$ pertencentes ao intervalo Eifeliano-Givetiano da Formação São Domingos, localizados no município de Tibagi, estado do Paraná, Brasil. O material está depositado no Laboratório de Estratigrafia e Paleontologia do Departamento de Geociências (DEGEO), da Universidade Estadual de Ponta Grossa (UEPG); sob prefixo DEGEO/MP. 
Todos os espécimes aqui referidos encontram-se preservados sob forma de impressões e compressões com cutícula preservada, sendo alguns compostos por partes e contrapartes (sensu SCHOPF, 1975). O estudo do material seguiu a metodologia padrão utilizada em Paleobotânica, como segue: técnica Dégagement (LECLERCQ, 1960) com auxílio de agulhas de aço, observação em microscópio estereoscópio, medições biométricas com paquímetro e régua. As fotografias foram realizadas com câmera Nikon D90 e lente macro 105 $\mathrm{mm}$, as ilustrações foram elaboradas com o uso do software Corel Draw X3.

\section{Aspectos taxonômicos da paleoflora da Formação São Domingos}

\subsection{Spongiophyton}

Incertae sedis

Família Spongiophytaceae Kräusel; Venkatachala (1966)

Gênero Spongiophyton Kräusel 1954 emend. Chaloner et al. (1974)

Espécie tipo Spongiophyton lenticulare (Barbosa) Kräusel 1954

Lâmina I, fig. A-I

Material: A. DEGEO/MP - 4972a; B. DEGEO/MP - 4947; C. DEGEO/MP - 4940; D. DEGEO/MP - 4995; E. DEGEO/MP - 4980; F. DEGEO/MP - 4772; G. DEGEO/MP 4932; H. DEGEO/MP - 4950; I. DEGEO/MP - 2832b.

Diagnose genérica emendada Chaloner et al. (1974, p.945):

"Tubular thallus with cuticular covering, branching dichotomously or subdichotomously with rounded apices; base unknown. Cuticle with internal cellular reticulum and circular to fusiform pores, largely confined to one surface of the thallus."

Descrição: Fragmentos do talo, preservados na forma de compressões fósseis com cutícula, que apresentam comprimento em torno de $25 \mathrm{~mm}$ e largura de $10 \mathrm{~mm}$. O talo com diferenciação dorsiventral é distinguido por duas superfícies, i.e. superfície "poral" ou dorsal e superfície "aporal" ou ventral. A primeira, de cutícula mais espessa, é recoberta por poros circulares a ovais de tamanho micrométrico, sem organização aparente; enquanto que a segunda apresenta cutícula mais fina, desprovida de poros, observando-se, por vezes, estrias longitudinais. As porções distais dos talos terminam em ápices arredondados ou afuniados, podendo, também, se ramificar dicotomicamente em mais de um plano. Estruturas reprodutivas são desconhecidas (Lâmina I, figs. A-I).

Discussão: Correspondem a plantas avasculares representadas por cutículas de talos achatados dorso-ventralmente, cobertas com poros distribuídos irregularmente na superfície dorsal. Abrange as espécies S. lenticularis, S. nanum, S. minutissimum e S. articulatum. Muitas hipóteses têm sido levantadas a respeito das afinidades paleobotânicas de Spongiophyton, seu hábito e habitat (GUERRA-SOMMER, 1993), tendo sido interpretado, ao longo do tempo, como animal colonial, alga, fungo, planta vascular ou briófita. Atualmente, Spongiophyton é incluído junto com os gêneros Aculeophyton e Orestovia na Família Spongiophytaceae (KRÄUSEL \& VENKATACHALA, 1966).

Portanto, apesar da abundância do gênero Spongiophy ton no registro fossilífero, a má preservação das estruturas anatômicas e a ausência de estruturas reprodutivas associadas 
têm levado a dúvidas quanto a sua afinidade botânica, a natureza de suas estruturas reprodutivas e se possuem ou não um sistema condutor verdadeiro (GENSEL et al., 1991). Stein et al. (1993) baseados em análises morfoanatômicas e estruturais consideraram os espongiófitos como um líquen. Esta concepção tem sido corroborada por alguns autores por meio de análises de Microscopia Eletrônica de Varredura (MEV) (TAYLOR et al., 2004) e de taxas de isótopos de carbono (JAHREN et al., 2003). Entretanto, Fletcher et al. (2004) demonstraram que os valores das taxas de isótopo de carbono em Spongiophyton não são significativamente diferentes dos valores de liquens e briófitas. Desta forma, as afinidades paleobotânicas de Spongiophyton permanecem conjecturais e mais estudos são necessários para esclarecer se este organismo possuiu ou não características intermediárias entre algas e plantas terrestres (TAYLOR et al., 2009).

Lâmina I. Spongiophyton sp. A. Superfície "poral", mostrando ramificação dicotômica (DEGEO/MP 4972a); B. Superfície "poral" do talo fragmentado (DEGEO/MP - 4947); C. Superfície "poral", mostrando talo com ápice afunilado (DEGEO/MP - 4940); D. Superfície "poral", mostrando talo com ramificação tripartite com ápices arredondados (DEGEO/MP - 4995); E. Superfície "poral" do talo fragmentado

(DEGEO/MP - 4980); F. Superfície "poral", mostrando talo com ramificação dicotômica com ápice arredondado (DEGEO/MP - 4772); G. Superfície "poral" do talo fragmentado (DEGEO/MP - 4932); H. Superfície "poral", mostrando ramificação dicotômica (DEGEO/MP - 4950); I. Superfície "poral" do talo fragmentado (DEGEO/MP - 2832b). Escala $5 \mathrm{~mm}$.

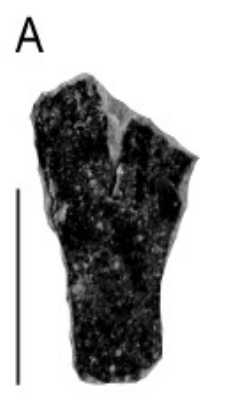

B
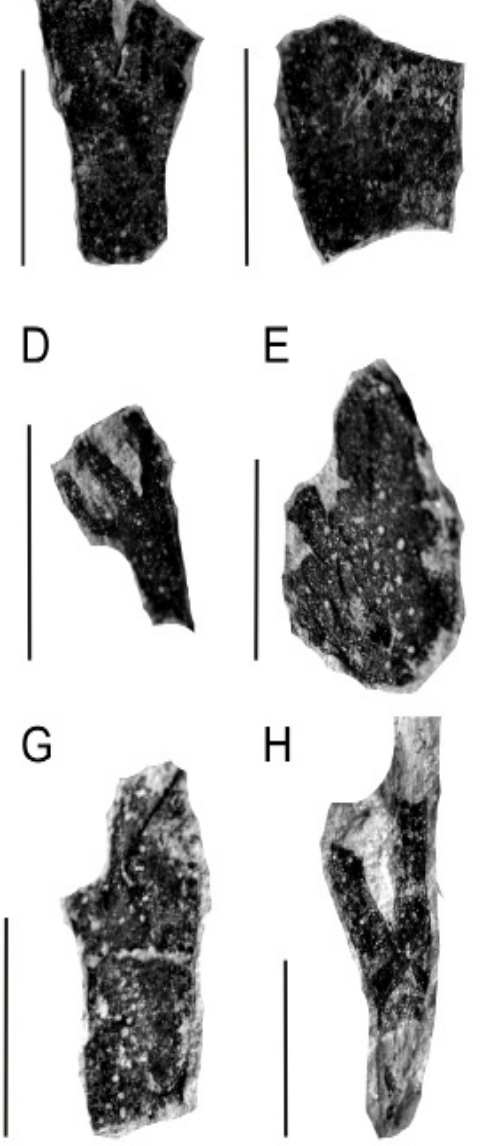

$\mathrm{E}$

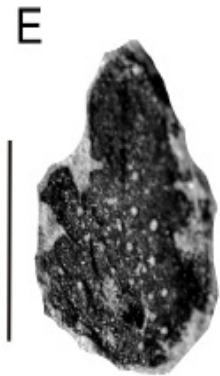

$\mathrm{H}$

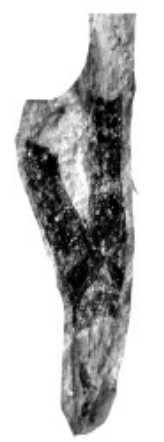

C

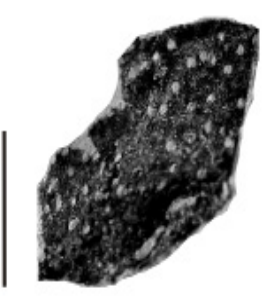

$\mathrm{F}$

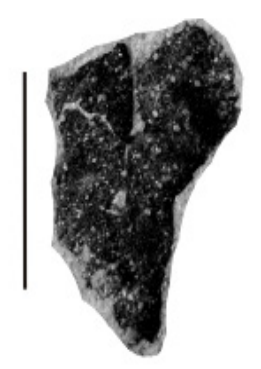

I

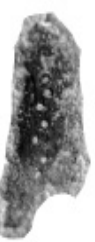




\subsection{Palaeostigma}

Classe Lycopsida

Incertae sedis

Gênero Palaeostigma Kräusel; Dolianiti, 1957

Espécie tipo Palaeostigma sewardii Kräusel; Dolianiti, 1957

Lâmina II, figuras A-I

Material: A. DEGEO/MP - 4946b; B. DEGEO/MP - 4979; C. DEGEO/MP - 4985; D. DEGEO/MP - 4984a; E. DEGEO/MP - 7288; F. DEGEO/MP - 4986; G. DEGEO/MP 3536; H. DEGEO/MP - 2830b; I. DEGEO/MP - 3632.

Diagnose genérica Kräusel; Dolianiti, (1957, p. 10):

"Caules, provavelmente, sem folhas verdadeiras, com numerosos apêndices espiniformes, rombudoconiformes, de arranjo irregular."

Descrição: Fragmentos de caules desfolhados, raramente bifurcados, preservados sob a forma de impressões e compressões fósseis com cutícula. Apresentam até $1,5 \mathrm{~cm}$ de comprimento e 1,0 cm de largura. A cutícula apresenta em sua superfície poros arredondados a alongados longitudinalmente e distribuídos de forma irregular ou apresentando ligeiro padrão helicoidal. Os poros representam apêndices espiniformes abscisados. Estruturas reprodutivas ou terminações do caule são desconhecidas (Lâmina II, figs. A-I).

Discussão: A espécie Palaeostigma sewardii foi descrita por Kräusel \& Dolianiti (1957) para designar restos vegetais ocorrentes nas camadas Picos, Formação Pimenteira, da Bacia do Parnaíba, incluindo ainda alguns espécimes atribuídos por Seward (1932) a Haplostigma irregularis (SEWARD, 1932, lâm. XXIII, figs. 1, 3, 4; lâm. XXIV, figs. 8, 9, 12; fig. text. 1), presentes nos grupos Bokkeveld e Witteberg na África do Sul. Devido a sua simplicidade morfológica e escassez no registro fóssil, Kräusel; Dolianiti (1957) classificaram o gênero como pertencente ao grupo das Psilophytas. Atualmente, o gênero é incluído dentro do grupo das licópsidas abrangendo três espécies, quais sejam: $P$. sewardii Kräusel \& Dolianiti, 1957, P. gracilis Anderson \& Anderson, 1985 e P. robusta Anderson \& Anderson, 1985.

Conforme apontado por Archangelsky et al. (1981), a pobre descrição de Palaeostigma levanta dúvidas quanto ao valor taxonômico dos caracteres mencionados na diagnose genérica. Desta forma, o aumento na amostragem poderá colaborar para o aprofundamento dos estudos sobre este fóssil.

Os trabalhos de campo recentemente executados em estratos do Devoniano Médio da Formação São Domingos (Bacia do Paraná) têm fornecido grande número de material fóssil que apresenta caracteres semelhantes ao gênero Palaeostigma. Estudos taxonômicos deste material poderão auxiliar na interpretação deste tipo de fóssil. 
Lâmina II. Palaeostigma sp. A. Fragmento do caule mostrando "poros" distribuídos irregularmente na superfície (DEGEO/MP - 4946b); B. Fragmento do caule mostrando "poros" distribuídos irregularmente na superfície (DEGEO/MP - 4979); C. Fragmento do caule mostrando "poros" com ligeira distribuição helicoidal (DEGEO/MP - 4985); D. Fragmento do caule mostrando "poros" distribuídos irregularmente na superfície (DEGEO/MP - 4984a); E. Fragmento do caule mostrando "poros" distribuídos irregularmente na superfície (DEGEO/MP - 7288); F. Fragmento do caule mostrando "poros" distribuídos irregularmente na superfície (DEGEO/MP - 4986); G. Impressão do caule mostrando contorno dos "poros" com ligeira distribuição helicoidal (DEGEO/MP - 3536); H. Impressão do caule mostrando contorno dos "poros" com ligeira distribuição helicoidal (DEGEO/MP - 2830b); I. Impressão do caule mostrando contorno dos "poros" com ligeira distribuição helicoidal (DEGEO/MP - 3632). Escala $10 \mathrm{~mm}$.

A

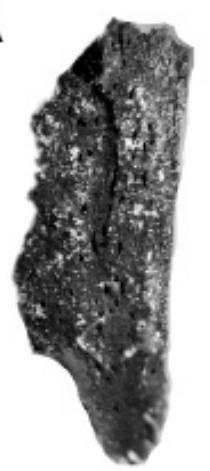

D

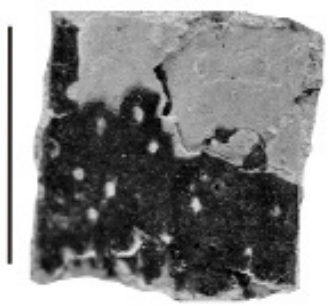

G

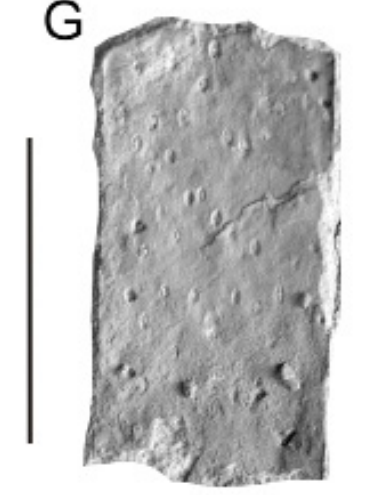

B

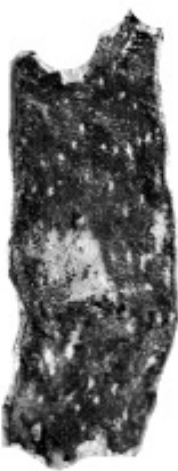

E

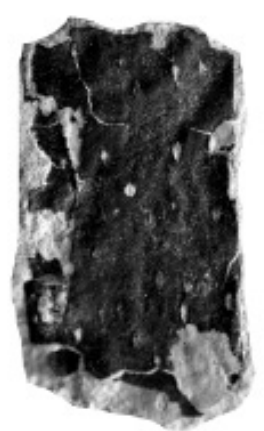

$\mathrm{H}$

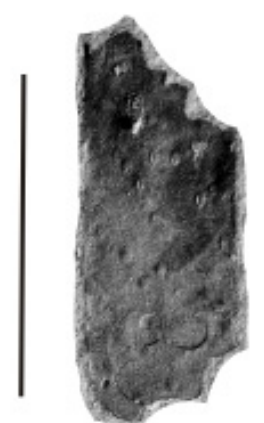

C

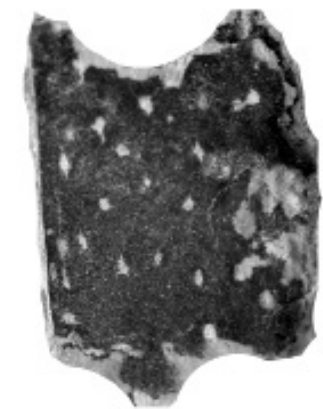

$\mathrm{F}$

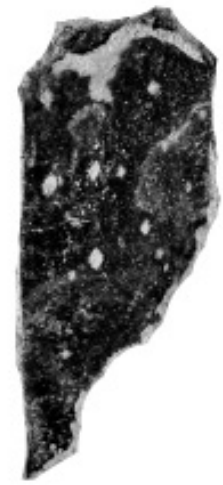

I

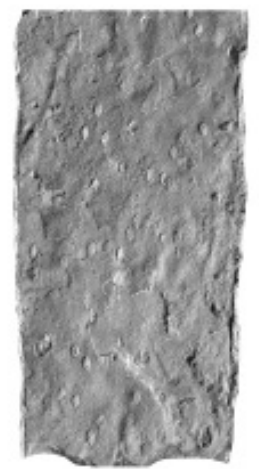

4.3 Haplostigma

Classe Lycopsida

Incertae sedis

Gênero Haplostigma Seward, 1932 emend. Anderson; Anderson 1985

Espécie tipo Haplostigma irregularis (Schwarz) Seward 1932

Lâmina 3, figuras A-I 
Material: DEGEO/MP - 7101; DEGEO/MP - 7152; DEGEO/MP - 4960a; DEGEO/ MP - 7233a; DEGEO/MP - 4974a; DEGEO/MP - 4987; DEGEO/MP - 7162; DEGEO/MP - 7499; DEGEO/MP - 4957.

Diagnose genérica emendada (SEWARD, 1932 emend. ANDERSON \& ANDERSON, 1985, p.89):

"Stems - erect fleshy to robust herbaceous lycopods, stems from 5-50 mm diam., very rarely forking, median vascular bundle not evident; leaf cushions not developed; leaf scars small to large, circular, less often longitudinally oval, spirally arranged, generally well-spaced; a central vascular trace (but no parichnos scars) sometimes evident."

Descrição: Fragmentos de caules preservados como impressões e compressões fósseis, de até $10 \mathrm{~cm}$ de comprimento e 1,0 cm de largura, raramente ramificados. Apresentam na superfície do caule falsas cicatrizes foliares com contorno arrendondado a ovalado longitudinalmente $(2,0 \times 3,0 \mathrm{~mm})$, dispostas em filotaxia helicoidal de baixo ângulo, num padrão lepidosigilarioide. A distância vertical entre as cicatrizes pode variar de 3-6 $\mathrm{mm}$, enquanto a distância lateral entre as cicatrizes adjacentes pode variar de 1-3 mm. Podem apresentar as bases foliares quebradas com contornos arredondados a alongados dependendo da posição da quebra. Traço foliar às vezes preservados (Lâmina III, figs. A-I).

Discussão: O gênero Haplostigma foi originalmente proposto para descrever impressões de caules ocorrentes nas camadas do Devoniano Médio-Superior da África do Sul (Grupos Bokkeveld e Witteberg), com a criação da espécie-tipo Haplostigma irregularis (SCHWARZ) SEWARD, 1932. Compreendem caules lisos de porte herbáceo, provavelmente bifurcado, portadores de almofadas foliares ovais a arredondadas em disposição helicoidal, com apêndices espinhosos rígidos (SEWARD, 1932 p. 365). Atualmente, Haplostigma inclui cinco espécies: H. irregularis, H. furquei FRENGUELLI 1952, H. lineare (WALKOM) MC LONGHLIN \& LONG 1994, H. baldisii GUTIÉRREZ \& ARCHANGELSKY, 1997 e H. kowiensis ANDERSON \& ANDERSON, 1985.

A pobre definição genérica do material tipo de Haplostigma, bem como pela reunião de três táxons diferentes (e.g. Palaeostigma, Drepanophycus e Haplostigma - KRÄUSEL \& DOLIANITI, 1957; PLUMSTEAD, 1967) têm levantado dúvidas quanto à diagnose genérica de Haplostigma. Mesmo com a emenda à diagnose genérica de Haplostigma proposta por Anderson \& Anderson (1985), com base na descrição de caules de três espécies (H. irregularis; $H$. kowiensis e $H$. furquei), ainda permaneceram dúvidas quanto a sua definição genérica.

Neste sentido, os autores têm utilizado o referido táxon como um morfogênero para caules de licófitas herbáceas com apêndices espiniformes ocorrentes no Devoniano do Gondwana (PLUMSTEAD, 1967; MCLOUGHLIN \& LONG, 1994; GUTIÉRREZ, 1996; GUTIÉRREZ \& ARCHANGELSKY, 1997; CINGOLANI et al., 2002; DI PASQUO et al., 2009; MOISAN et al., 2011), transformando-o em um saco de gatos taxonômico. 
Lâmina III. Haplostigma irregularis. A. Molde externo do caule apresentando as cicatrizes foliares em filotaxia helicoidal (DEGEO/MP - 7101); B. Fragmento do caule (DEGEO/MP - 7152); C. Fragmento do caule com as cicatrizes equidistantemente separadas (DEGEO/MP - 4960a); D. Molde interno do caule apresentando as cicatrizes foliares em filotaxia helicoidal, a distância vertical entre as cicatrizes é maior que a distância horizontal (DEGEO/MP - 7233a); E. Fragmento do caule com as cicatrizes equidistantes (DEGEO/MP - 4974a); F. Fragmento do caule apresentando as cicatrizes foliares em filotaxia helicoidal, a distância vertical entre as cicatrizes é maior que a distância horizontal (DEGEO/MP - 4987); G. Fragmento do caule apresentando as cicatrizes foliares em filotaxia helicoidal (DEGEO/MP - 7162); H. Fragmento do caule apresentando as cicatrizes foliares em filotaxia helicoidal (DEGEO/MP - 7499); I. Fragmento do caule apresentando as cicatrizes foliares em filotaxia helicoidal, a distância horizontal entre as cicatrizes é maior que a distância vertical (DEGEO/MP - 4957). Escala $10 \mathrm{~mm}$.

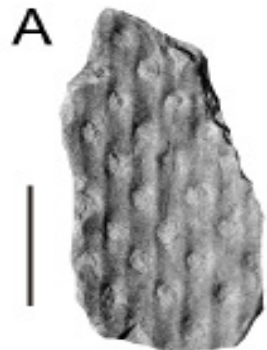

D

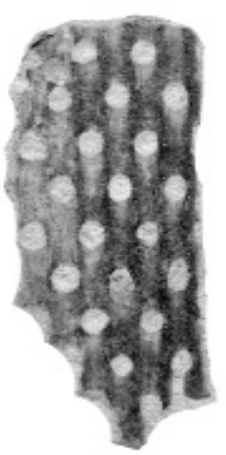

G

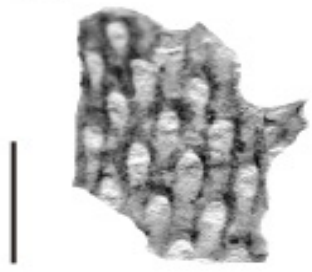

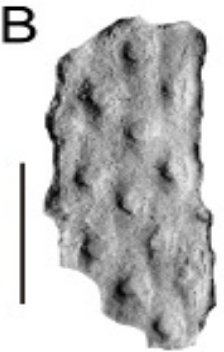
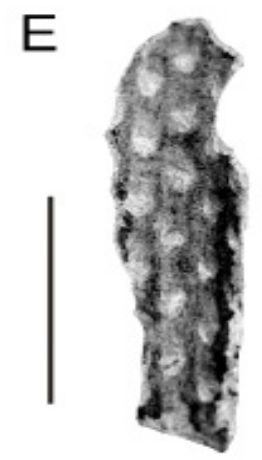

$\mathrm{H}$

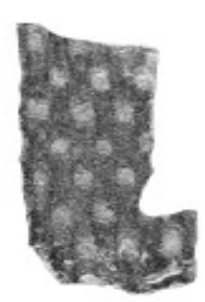

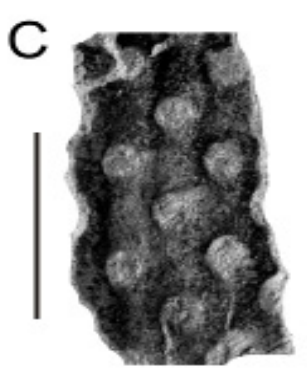

$\mathrm{F}$

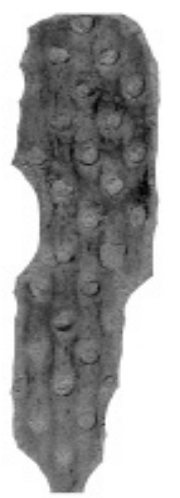

I

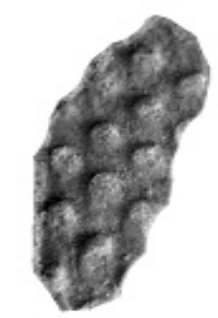

\section{NOVOS ACHADOS E PERSPECTIVAS FUTURAS}

Com exceção das plantas vasculares ocorrentes no topo da Formação Furnas, os estudos com o material paleobotânico ocorrente nas formações Ponta Grossa e São Domingos ficaram, por muito tempo, postergados e diluídos na literatura. Nos últimos anos, a execução de trabalhos de campo no município de Tibagi-PR tem possibilitado a prospecção de fitofósseis bem preservados, resgatando o interesse científico da paleobotânica nestas unidades, principalmente na Formação São Domingos. 
Foram identificados e descritos três táxons ocorrentes nos afloramentos Sítio Wolff e Itáytyba (Formação São Domingos, Devoniano Médio), a saber: Spongiophyton, Palaeostigma e Haplostigma.

As análises tafonômicas e sedimentológicas efetuadas com a macropaleofauna de invertebrados marinhos bentônicos aliadas aos dados de estratigrafia de sequências têm proporcionado novas interpretações sobre os sistemas deposicionais e paleoambientais do Devoniano do estado do Paraná (BOSETTI et al., 2009; 2011; 2012).

A utilização destas ferramentas no estudo dos restos vegetais relatados em Bosetti et al. (2011) e Matsumura et al. (2010; 2011) nos estratos devonianos da Bacia do Paraná, no estado homônimo, em conjunto aos restos vegetais de outras bacias sedimentares brasileiras (Bacia do Parnaíba e Bacia do Amazonas), bem como, os registros do Gondwana Ocidental (Argentina; Bolívia; Colômbia; Chile; Uruguai e Venezuela) e Gondwana Oriental (Austrália e Antártica), vêm a colaborar na compreensão nos estudos evolutivos e paleoecológicos das plantas, além de fornecer dados relevantes para interpretações dos sistemas deposicionais e reconstituições paleofitogeográficas.

\section{AGRADECIMENTOS}

Os autores agradecem ao Conselho Nacional de Desenvolvimento Científico e Tecnológico (CNPq), pelo apoio por meio da concessão de bolsas (Processo 141979/2011-9; 305687/2010-7) e auxílios (processos 401796/2010-8; 479474/2011-0), fundamentais para a realização deste trabalho e a Dra . Mercedes Di Pasquo (CICYTTP/CONICET) pela revisão do manuscrito.

\section{REFERÊNCIAS}

ANDERSON, John M.; ANDERSON, Heidi M. Palaeofloras of Southern Africa. Prodromus of South Africa megafloras, Devonian to Lower Cretaceous. Rotterdam, Balkema, 1985.

ARCHANGELSKY, Sergio; AZCUY, Carlos L.; WAGNER, R.H. Three dwarflycophytes from the Carboniferous of Argentina. Scripta Geologica, n. 64, 1981, p. 1-35.

BALDIS, Bruno A.J. Paleozoogeografía de los trilobites devónicos en Sudamérica Austral. Ameghiniana, v. 16, 1979, p. 209-216.

BARBOSA, Octavio. Vegetais fósseis do devoniano do Brasil e da Bolívia. Mineração e Metalurgia, n. 15, v. 81, 1949, p. 81-84.

BERRY, Christopher M.; EDWARDS, Dianne. New species of the Devonian lycophyte Colpodexylon from the Devonian of Venezuela. Palaeontographica B, n. 237, 1995, p. 59-74.

BERRY, Christopher M.; MOREL, Eduardo; MOJICA, Jairo; VILLARROEL, Carlos. Devonian plants from Colombia, with discussion of their geological and palaeogeographical context. Geological Magazine, n. 137, 2000, p. 257-268.

BIGARELLA, João José; SALAMUNI, Riad; MARQUES FILHO, Pedro Lagos. Estruturas e texturas da Formação Furnas e sua significação paleogeográfica. Boletim da Universidade Federal do Paraná. Curitiba, Geologia, n. 18, 1966, p. 1-114. 
BOLZON, Robson Tadeu; ASSINE, Mário Luis; GUERRA SOMMER, Margot. Ocorrência de Cooksonia Lang, 1937 na Formação Furnas, Estado do Paraná. VIII REUNIÃO DE PALEOBOTÂNICOS E PALINÓLOGOS, 1994, São Paulo. Anais de resumo, USP, p. 11.

BOLZON, Robson Tadeu; ASSINE, Mário Luis; GUERRA SOMMER, Margot. Ocorrência de Cooksonia Lang, 1937 na Formação Furnas, Estado do Paraná. In: VIII REUNIÃO DE PALEOBOTÂNICOS E PALINÓLOGOS, 1994, São Paulo. Anais de resumo, USP, p. 11.

BOSETTI, Elvio Pinto. Tafonomia de alta resolução das fácies de offshore da sucessão devoniana da região de Ponta Grossa - Paraná, Brasil. 2004. Tese (Doutorado em Geociências) - Programa de Pós-Graduação em Geociências, UFRGS, Porto Alegre-RS.

BOSETTI, Elvio Pinto; PEYERL, Drielli; HORODYSKI, Rodrigo Scalise; ZABINI, Carolina. Formação Ponta Grossa: História, Fácies e Fósseis. I SIMPÓSIO DE PESQUISA EM ENSINO E HISTÓRIA DE CIÊNCIAS DA TERRA E III SIMPÓSIO NACIONAL SOBRE ENSINO DE GEOLOGIA NO BRASIL, 2007, Campinas. Anais de resumo, UNICAMP, 2007, p. 353-360.

BOSETTI, Elvio Pinto; GODOY, Luiz Carlos; MYSINSKI JUNIOR, Lucinei José, HORODYSKI, Rodrigo Scalise; ZABINI, Carolina. Interpretação paleoambiental na sequência basal da formação Ponta Grossa (devoniano) do município de Ponta Grossa, Paraná, Brasil. Terr@Plural, Ponta Grossa, v.3, n.1, jan./jul. 2009, p.137-156.

BOSETTI, Elvio Pinto; GRAHN, Yngve; HORODYSKI, Rodrigo Scalise; MENDLOWICZ MAULLER, Paula; BREUER, Pierre; ZABINI, Carolina. An earliest Givetian "Lilliput Effect" in the Paraná Basin, and the collapse of the Malvinokaffric shelly fauna. Paläontologische Zeitschrift, v. 85, n. 1, mar. 2011, p. 49-65.

BOSETTI, Elvio Pinto; GRAHN, Yngve; HORODYSKI, Rodrigo Scalise; MENDLOWICZ MAULLER, Paula. The first recorded decline of the Malvinokaffric Devonian fauna in the Paraná Basin (southern Brazil) and its cause; taphonomic and fossil evidences. Journal of South American Earth Sciences, n. 37, ago. 2012, p. 228-241.

BOUCOT, Arthur J. Malvinokaffric Devonian marine comunity distribution ciencias. Anais da Academia Brasileira de Ciências, n. 43(suplemento), 1971, p. 23-49.

BOUCOT, Arthur J.; GILL, E.D. Australocoelia, a new Lower Devonian Brachiopod from South Africa, South America and Australia. Journal of Paleontology, v. 30, n. 5, p.1173-1178, sep. 1956.

BOUREAU, M.E.; PONS, D. Sur des empreintes végétales devoniènnes du Sud de La Bolivie. Compte Rendus de l'Académie Sciences de Paris, Série D, n. 276, 1973, p. 2151-2153.

CHALONER, W.G.; MENSAH, M.K.; CRANE, M.D. Non-vascular land plants from the Devonian of Ghana. Palaeontology, v. 17, n. 4, 1974, p. 925-947.

CINGOLANI, Carlos A.; BERRY, Christopher M.; MOREL, Eduardo; TOMEZZOLI, Renata. Middle Devonian lycopsids from high southern palaeolatitudes of Gondwana (Argentina). Geological Magazine, v. 139, n. 6, nov. 2002, p. 641-649.

CLARKE, John Mason. Fósseis Devonianos do Paraná. Monographias do Serviço Geológico e Mineralógico do Brasil. Rio de Janeiro, 1913.

Di PASQUO, Mercedes; NOETINGER, Sol. First record of Early Devonian (Lochkovian) flora from the Santa Rosa Formation - Alarache, Southern Bolivia. Geologica Acta, v.6, n. 2, Jun. 2008, p. 191-210.

Di PASQUO, Mercedes; AMENABAR, Cecília Rodriguez; NOETINGER, Sol. Middle Devonian microfloras and megafloras from western Argentina and southern Bolivia: their importance in the palaeobiogeographical and palaeoclimatic evolution of western Gondwana. In: KONIGSHOF, Peter (Ed.). Devonian change: case studies in palaeogeography and palaeoecology. The Geological Society, London, Special Publications, n. 314, 2009, p. 193-213.

EDWARDS, Dianne. Constraints on Silurian and Early Devonian phytogeographic analysis based on megafossils. In: McKERROW, William S.; SCOTESE, Christopher R. (Eds). Paleozoic Paleogeography and Biogeography. Geol. Soc. London Mem., 12 ,1990, p. 233-242.

FLETCHER, Benjamin J.; BEERLING, David J.; CHALONER, William G. Stable carbon isotopes and the metabolism of the terrestrial Devonian organism Spongiophyton. Geobiology, n. 2, 2004, p. 107-119. 
FRENGUELLI, Joaquin. Haplostigma furquei n. sp. del Devónico de la Precordillera de San Juan. Revista de la Asociación Geológica Argentina, v. 7, n. 1, 1952, p. 5-10.

GAUGRIS, Kariny de Amorim; GRAHN, Yngve. New chitinozoan species from the Devonian of the Paraná Basin, south Brazil, and their biostratigraphic significance. Ameghiniana, v. 43, n. 2, 2006, p. 293-310.

GENSEL, Patricia G.; CHALONER, William G.; FORBES, William H. Spongiophyton from de Late lower Devonian of New Brunswick and Quebec, Canada. Palaeontology, v. 34, n. 1, 1991, p. 149-168.

GERRIENNE, Philippe; FAIRON-DEMARET, Muriel; GALTIER, Jean; LARDEUX, Hubert; MEYERBERTHAUD, Brigitte; RÉGNAULT, Serge; STEEMANS, Philippe. Plants Associated with Tentaculites in a New Early Devonian Locality from Morocco. Abhandlungen der Geologischen Bundesanstalt, n. 54, 1999, p. 323335.

GERRIENNE, Philippe; BERGAMASHI, Sérgio; PEREIRA, Egberto; RODRIGUES, Maria Antonieta da Conceição; STEEMANS, Philippe. An Early Devonian flora, including Cooksonia, from the Paraná Basin (Brazil). Review of Palaeobotany and Palynology, v. 116, n. 1-2, 2001, p. 19-38.

GERRIENNE, Philippe; DILCHER, David L.; BERGAMASCHI, Sérgio; MILAGRES, Ingrid; PEREIRA, Egberto; RODRIGUES, Maria Antonieta da Conceição. An exceptional specimen of the early land plant Cooksonia paranensis, and a hypothesis on the life cycle of the earliest eutracheophytes. Review of Palaeobotany and Palynology, v. 142, n. 3-4, 2006, p. 123-130.

GHILARDI, Renato Pirani. Tafonomia comparada e paleoecologia dos macroinvertebrados (ênfaseem trilobites), da Formação Ponta Grossa (Devoniano, Sub-bacia Apucarana), Estado do Paraná, Brasil. 2004. Tese (Doutorado em Geologia Sedimentar) - Programa de Pós-Graduação em Geologia Sedimentar, USP, São Paulo-SP.

GONEZ, Paul; GERRIENNE, Philippe. Aberlemnia caledonica gen. et comb. nov., a new name for Cooksonia caledonica Edwards 1970. Review of Palaeobotany and Palynology, v. 163, n. 1-2, 2010, p. 64-72.

GRAHN, Yngve; MENDLOWICZ MAULLER, Paula; BERGAMASCHI, Sérgio; BOSETTI, Elvio Pinto. Palynology and sequence stratigraphy of three Devonian rock units in the Apucarana Sub-basin (Paraná Basin, south Brazil): Additional data and correlation. Review of Paleobotany and Palynology. http://dx.doi. org/10.1016/j.revpalbo.2011.10.006.

GUERRA-SOMMER, Margot. Spongiophyton nas Bacias Intracratônicas Brasileiras: Considerações Paleoecológicas e Bioestratigráficas. Pesquisas em Geociências, v. 20, n. 1, 1993, p. 70-77.

GUERRA-SOMMER, Margot; DIAS-FABRICIO, Maria Elice; CAZZULO-KLEPZIG, Miriam; MARQUESTOIGO, Marleni; PILATTI, Fernando. Spongiophyton and associated micro and megafossils from the Devonian of the Ponta Grossa Formation, Paraná Basin, South Brazil. VII REUNIÃO DE PALEOBOTÂNICOS E PALINÓLOGOS, 1990, São Paulo. Anais de resumo. USP, p. 22.

GUERRA-SOMMER, Margot; RODRIGUES, René; MENDONÇA FILHO, João Graciano; MARQUES-TOIGO, Marleni; CAZZULO-KLEPZIG, Miriam; PILATTI, Fernando. Characterization of Spongiophyton Habit Using Morphologic Description, Organic Geochemistry and Organic Petrography. I SIMPÓSIO SUL-AMERICANO DO SILURO-DEVONIANO, 1996, Ponta Grossa. Anais de resumo. UEPG, p. 107-116.

GUTIERREZ, Pedro Raúl. Revisión de las licopsidas de la Argentina 2. Malanzania Archangelsky, Azcuy et Wagner y Haplostigma Seward; con notas sobre Cyclostigma Haughton. Ameghiniana, v. 32, n. 29, 1996, p. 127-144.

GUTIERREZ, Pedro Raúl; ARCHANGELSKY, Sérgio. Haplostigma baldisii sp. nov. (Lycophyta) del Devónico de La Precordillera de San Juan, Argentina. Ameghiniana, v. 34, n. 3, 1997. p. 275-282.

JAHREN, A. Hope; PORTER, Steven; KUGLITSCH, Jeffrey J. Lichen metabolism identified in Early Devonian terrestrial organisms. Geology, v. 31, n. 2, fev. 2003, p. 99-102.

KRÄUSEL, Richard. Spongyophyton nov. gen. (Tallophyta) und Haplostigma Seward (Pteridophyta) im unterDevon von Paraná. LANGE, FredericoWaldemar (org) Paleontologia do Paraná. Curitiba: Comissão de Comemorações do Centenário do Paraná, 1954, p. 195-210.

KRÄUSEL, Richard; DOLIANITI, Elias. Restos vegetais das Camadas Picos, Devoniano Inferior do Piauí. Boletim do Ministério da Agricultura. Departamento Nacional da Produção Mineral, Divisão de Geologia e Mineralogia, n. 173, 1957, p. 7-19. 
KRÄUSEL, Richard; VENKATACHALA, B.S. Devonische Spongiophytaceen aus Destnud. West-Asien. Senkenbergiana Lethaia, n. 47, 1966, p. 215-251.

LECLERCQ, S uzanne. Refendage d'une roche fossilifère et dégagement de ses fossiles sous binoculaire. Senckenbergiana Lethaea, n. 41, 1960, p. 483-487.

MACHADO, Luciano Gandin; BOLZON, Robson Tadeu; AZEVEDO, Inês; CARVALHO, Marcelo de Araújo; SOUZA, Alice Ferreira. Primeiras plantas terrestres no Eodevoniano (Formação Furnas) do estado do Paraná, Brasil. CARVALHO, Ismar de Souza; RODRIGUES, Maria A. Conceição; CASSAB, Rita de Cássia Tardin (Orgs.) Paleontologia - Cenários de Vida. Rio de Janeiro: Interciência, v. 1, 2007, p. 49-59.

MATSUMURA, Willian Mikio Kurita; IANNUZZI, Roberto; BOSETTI, Elvio Pinto; MARCONDES, Andrea Thays Paganella. Novos registros paleobotânicos da Formação São Domingos, Devoniano do Estado do Paraná. XII REUNIÃO REGIONAL DA SOCIEDADE BRASILEIRA DE PALEONTOLOGIA (PALEO PR/ SC), 2010, Cascavel. Anais de resumo, UNIOESTE, p.12.

MATSUMURA, Willian Mikio Kurita; IANNUZZI, Roberto; BOSETTI, Elvio Pinto. Registro Paleobotânico do Devoniano do Paraná: Aplicações bioestratigráficas e implicações paleofitogeográficas. XIII REUNIÃO ANUAL DA SOCIEDADE BRASILEIRA DE PALEONTOLOGIA (PALEO PR/SC), 2011, Mafra. Anais de resumo, UNC, p. 19.

MATSUMURA, Willian Mikio Kurita; PINHEIRO, Esther Regina S.; IANNUZZI, Roberto; BOSETTI, Elvio Pinto. Registro de Interações Planta-Arthropoda no Devoniano Médio da Bacia do Paraná. I SIMPÓSIO BRASILEIRO DE PALEOINVERTEBRADOS, 2012, Bauru. Boletim Informativo da Sociedade Brasileira de Paleontologia - Edição Especial. UNESP - BAURU, p. 52.

MATSUMURA, Willian Mikio Kurita; BOSETTI, Elvio Pinto; IANNUZZI, Roberto. Estudo tafonômico das plantas fósseis do Devoniano Médio do estado do Paraná, Brasil. XIV SIMPÓSIO BRASILEIRO De PALEOBOTÂNICA E PALINOLOGIA, 2013, Rio de Janeiro. Anais do Museu Nacional - UFRJ, p. 109.

McLOUGHLIN, Stephen; LONG, John A. New records of Devonian plants from southern Victoria Land, Antarctica. Geological Magazine, v. 131, n. 1, 1994, p. 81-90.

MELO, José Henrique Gonçalves. The Malvinokaffric Realm in the Devonian of Brazil. In: McMILLAN, N.J.; EMBRY, A.F.; GLASS, D.J. (Eds.) Devonian of the World. Canadian Society of Petroleum Geologists, Proceedings of the $2^{\text {nd }}$ International Symposium on the Devonian System. Calgary,C.S.P.G., 1988 Memoir, 14 (1), p. 667-703.

MENDLOWICZMAULLER, Paula; GRAHN, Yngve; CARDOSO, Tereza Regina Machado. Palynostratigraphy from the Lower Devonian of the Paraná Basin, south Brazil, and a revision of contemporary chitinozoan biozones from western Gondwana. Stratigraphy, v. 6, 2009, p. 313-332.

MILAGRES, Ingrid de Melo; PEREIRA, Egberto; BERGAMASCHI, Sérgio; RODRIGUES, Maria Antonieta da Conceição; GERRIENNE, Philippe. O registro da tafoflora devoniana observada no arco de Ponta Grossa, Bacia do Paraná, e suas implicações paleogeográficas. CARVALHO, Ismar de Souza; RODRIGUES, Maria A. Conceição; CASSAB, Rita de Cássia Tardin (Orgs.) Paleontologia - Cenários de Vida. Rio de Janeiro: Interciência, v. 1, 2007, p. 25-48p.

MOISAN, Philippe; NIEMEYER, Hans; KERP, Hans. Lycopsids from the Upper Devonian of northern Chile with remarks on the geographical distribution of the morphogenus Haplostigma Seward. Paläontologische Zeitschrift, v. 85, n. 3, set. 2011, p 231-240.

MUSSA, Diana; BORGHI, Leonardo. A presença do gênero Horneophyton BARGHOORN; DARRAH na Formação Furnas, bacia do Paraná. Anais da Academia Brasileira de Ciências, v. 65, n. 2, 1993, p. 214-215.

MUSSA, Diana; BORGHI, Leonardo; BERGAMASCHI, Sérgio; SCHUBERT, G.; PEREIRA, Egberto; RODRIGUES, Maria Antonieta da Conceição. Estudo preliminar da tafoflora da Formação Furnas, Bacia do Paraná, Brasil. Anais da Academia Brasileira de Ciências, v. 68, n. 1, 1996, p. 65-89.

MUSSA, Diana; BORGHI, Leonardo; BERGAMASCHI, Sérgio; SCHUBERT, G.; PEREIRA, Egberto; RODRIGUES, Maria Antonieta da Conceição; PEREIRA, J.F.; EMMERICH, M. New Taxa From Furnas 
Formation, Paraná Basin, Brasil - An Approach and Revalidation of Names. Boletim do Herbarium Bradeanum, v. 45, n. 7, 2002, p. 303-309.

OPPENHEIM, Victor. Fósseis devonianos do Paraná. Anais da Academia Brasileira de Ciências, v. 7, n. 4, 1935,p. 345-348.

PETRI, Setembrino. Contribuição ao estudo do Devoniano Paranaense. Boletim da Divisão de Geologia e Mineralogia, n. 129, 1948, p. 1-125.

PLUMSTEAD, Edna Pauline. A general review of the Devonian fossil plants found in the Cape System of South Africa. Palaeontologia Africana, n. 10, 1967, p. 1-83.

QUADROS, Raquel. Paleontologia dos Brachiopoda - Lingulida, Strophomenida, Spiriferida, Terebratulida - Devoniano, da Serra de Atimã e arredores, Mato Grosso - Brasil. 1987. Tese (Doutorado em Geociências) Programa de Pós-Graduação em Geociências, UFRGS, Porto Alegre-RS.

RACHEBOEUF, Patrick R.; CASIER, Jean-Ggeorges; PLUSQUELLEC, Yves; TORO, Margarita; MENDOZA, David; PIRES DE CARVALHO, Maria da Gloria; LE HÉRISSÉ, Alain; PARIS, Florentin; FERNÁNDEZMARTÍNEZ, Esperanza; TOURNEUR, Francis; BROUTIN, Jean; CRASQUIN, Sylvie; JANVIER, Philippe. New data on the Silurian-Devonian palaeontology and biostratigraphy of Bolivia. Bulletin of Geosciences, v. 87, n. 2, 2012, p. 269-314.

RAYMOND, Anne; PARKER, William C.; BARRETT, Stephen. F. Phytogeography of the Early Devonian. In: TIFFNEY, Bruce (ed.) Geological Factors and the Evolution of Plants. Yale University Press, New Haven, 1985, p. 129-167.

RAYMOND, Anne. Paleogeographic distribution of Early Devonian plant traits. Palaios, v. 2, n. 2, abr. 1987, p. 113-132.

READ, Charles B. Plantas fósseis do Neopaleozóico do Paraná e Santa Catarina. Div. Geol. Miner., DNPM, Monog. n. 12, 1941, p. 1-102.

RODRIGUES, Maria Antonieta da Conceição; PEREIRA, Egberto; BERGAMASCHI, Sérgio. Ocorrência de psilophytales na formação Furnas, bordo leste da Bacia do Paraná. Boletim IG-USP. Série Científica, São Paulo, v. 7, 1989, p. 35-43.

RODRIGUES, Sabrina Coelho. Tafonomia comparada dos conulatae Collins et al 2000, Formação Ponta Grossa, Devoniano (?Lochkoviano-Frasniano, Bacia do Paraná: Implicações Paleoautoecológicas e Paleoambientais. 2002. Dissertação (Mestrado em Geologia Sedimentar) - Programa de Pós-Graduação em Geologia Sedimentar, USP, São Paulo-SP.

RODRIGUES, Maria Antonieta da Conceição; SOARES, Hilda Leonor Cuevas de Azevedo; BERGAMASCHI, Sérgio. Horizontes de Mortalidade em Massa de Tentaculita na Formação Ponta Grossa, Bacia do Paraná. In: MELO, José Henrique Gonçalves; TERRA, G.J.S. (Eds.), Correlação de Sequências Paleozóicas Sul-Americanas. Ciência - Técnica - Petróleo. Seção: Exploração de Petróleo v. 20. p. 73-79.

SCHEFFLER, Sandro Marcelo. Morfótipos de pedúnculo de pelmatozoários da Formação Ponta Grossa (Devoniano, Bacia do Paraná), no Estado do Paraná, Brasil. Terr@ Plural, v. 1, n. 2, ago.-dez., 2007, p. 139-151.

SCHEFFLER, Sandro Marcelo. Crinoides e Blastoides do Devoniano brasileiro. 2010. Tese (Doutorado em Geologia) - Programa de Pós-Graduação em Geologia, UFRJ, Rio de Janeiro-RJ.

SCHEFFLER, Sandro Marcelo; FERNANDES, Antônio Carlos Sequeira. Crinoidea da Formação Ponta Grossa (Devoniano, Bacia do Paraná), Brasil. Arquivos do Museu Nacional, v. 65, n. 1, 2007a, p. 83-98.

SCHEFFLER, Sandro Marcelo; FERNANDES, Antônio Carlos Sequeira. Blastoidea da Formação Ponta Grossa (Devoniano, Bacia do Paraná), Estado do Paraná, Brasil. Arquivos do Museu Nacional, v. 65, n. 1, 2007b, p. 99-112.

SCHOPF, James M. Modes of fossil preservation. Review of Palaeobotany and Palynology, v. 20, n. 1-2, 1975, p. 27-53.

SEWARD, Albert Charles. Fossil plants from the Bokkeveld and Witteberg beds of South Africa. Quartely Journal of the Geological Society of London, v. 88, 1932, p. 358-369. 
SIMÕES, Marcello Guimarães; SALES, Alexandre Magno Feitosa; GHILARDI, Renato Pirani; HOLZ, Michael; RODRIGUES, Sabrina Coelho; LEME, Juliana de Moraes. Assinaturas tafonômicas como marcadoras de limites de parasequências em offshore settings: um exemplo do Devoniano, Bacia do Paraná, Brasil. XLI CONGRESSO BRASILEIRO DE GEOLOGIA, 2002, João Pessoa, Anais de resumo, p. 680-681.

SIMÕES, Marcello Guimarães; RODRIGUES, Sabrina Coelho; LEME, Juliana de Moraes; ITEN, Heyo Van. Some Middle Paleozoic Conulariids (Cnidaria) as Possible Examples of Taphonomic Artifacts. Journal of Taphonomy, v. 1, n. 3, 2003, p.165-186.

SOMMER, Friedrich Wilhelm. Contribuição a Paleofitogeografia do Paraná. In: LANGE, Frederico Waldemar (Ed.) Paleontologia do Paraná. Comissão de Comemorações do Centenário do Paraná, Curitiba, 175-194 p.

SPRECHMANN, Peter; MONTAÑA, J.; GAUCHER, Claudio. Devónico. In: Geología y recursos minerales del departamento de Durazno. Edición de la intendencia municipal de Durazno, 25-55, 1993.

STEIN, William E.; HARMON, Gary D.; HUEBER, Francis M. Spongiophyton from the Lower Devonian of North America reinterpreted as a lichen. American Journal of Botany, v. 80, n. 6, 1993, p.80-93.

TAYLOR, Wilson A.; FREE, Chris, BOYCE, Carolyn, HELGEMO, Rick; OCHOADA, Jaime. SEM analysis of Spongiophyton interpreted as a fossil lichen. International Journal of Plant Sciences, v. 165, n. 5, 2004, p. 875881.

TAYLOR, Thomas, N.; TAYLOR, Edith L.; KRINGS, Michael. The Biology and Evolution of Fossil Plants. 2.ed. Burlington, MA, London, UK, San Diego, CA, New York NY, Academic Press, 2009.

WNUK, Christopher. The development of floristic provinciality during the Middle and Late Paleozoic. Review of Palaeobotany and Palynology, v. 90, n. 1-2, 1996.

ZABINI, Carolina; BOSETTI, Elvio Pinto; HOLZ, Michael. Taphonomy and taphofacies analysis of lingulid brachiopods from Devonian sequences of the Paraná Basin, Brazil. Palaeogeography, Palaeoclimatology, Palaeoecology, v. 292, n. 1-2, 2010, p. 44-56.

ZABINI, Carolina; HOLZ, Michael; BOSETTI, Elvio Pinto; MATSUMURA, Willian Mikio Kurita; HORODYSKI, Rodrigo Scalise. Sequence stratigraphy and taphonomic signatures of marine invertebrates: A Devonian (Pragian/Eifelian) example of the Paraná Basin, Brazil. Journal of South American Earth Sciences, v. 33, n. 1, 2012, p. 8-20.

ZDEBSKA, Danuta. On Spongiophyton from the Lower Devonian of Poland.

Recebido em 19/07/2013

Aceito para publicação em 02/12/2013 